\title{
Towards automatic defect detection in carbon fiber composites using active thermography
}

\author{
by R. Usamentiaga*, P. Venegas ${ }^{* *}$, J. Guerediaga** and L. Vega** \\ * *Department of Computer Engineering, University of Oviedo, Campus de Viesques, 33204 Gijón, Spain, \\ rusamentiaga@uniovi.es \\ ** Centro de Tecnologías Aeronáuticas (CTA), Parque Tecnológico de Álava, Juan de la Cierva 1, \\ 01510 Miñano, Spain, pvenegas@ctaero.com
}

\begin{abstract}
Major advances have occurred in the field of non-destructive testing based on active thermography. However, active thermography has yet to be integrated in the automation of a production line for defect detection. There is a general agreement about stimulation and post-processing methods that improve defect detectability, however, a general framework for automatic defect detection has not been defined. This work presents a general framework towards automatic defect detection using active thermography. The proposed framework has four steps: acquisition, enhancement, filtering and segmentation. The most appropriate methods are selected for each step. Finally, the performance of the proposed method is evaluated using a real carbon fiber composite.
\end{abstract}

\section{Introduction}

Safety is a major requirement in the aerospace industry. As a result, product inspection and quality control are of utmost importance. Product inspection is not only essential for the safe operation of aircraft components designed to perform for decades, but it also results in better products and higher revenue [1].

One of the most successful technologies used to inspect aeronautical products is active infrared thermography [2]. This technology is particularly advantageous for defect detection, as it can be applied very quickly to the inspection of materials for subsurface defect detection. Also, active thermography is a clean and non-intrusive technology, which has been proven successful for non-destructive testing [3]. In active infrared thermography an external stimulus is applied to the source of the inspected specimen causing non-stationary heat flow. The propagation of heat depends on many material properties, such as thermal conductivity or density, but also on subsurface anomalies which result in temperature differences on the surface target [4].

Traditionally, product inspection in quality control is performed by human experts [5]. However, humans provide subjective feedback based on their skills and training, and are unable to extract precise information in quick or repetitive tasks. Moreover, in infrared thermography thermal contrast between regions of interest may appear as subtle signatures due to many factors that degrade thermographic data, such as signal noise and the uneven properties of the surface of the material being inspected. In this context, product inspection based on raw infrared images is not appropriate and infrared image processing is required [6].

Automatic product inspection requires robust image processing algorithms. Generally, image processing algorithms are broken down into several steps, each one dealing with a different abstraction of the problem. The first steps deal with acquisition and filtering; the last steps with pattern recognition and classification. In recent decades, major advances have occurred in each of these steps, and many image analysis routines have been proposed. In general, there is agreement about the required steps and the most appropriate methods for each of them in different type of applications [7, 8]. Research has produced many commercial applications available for automated visual inspection [9]. However, in the case of defect detection using active thermography, no agreement has been reached about the steps themselves, much less about the most adequate methods for each step.

This work presents a general framework towards automatic defect detection using active thermography. The proposed framework is based on a multi-step process, similar to most image processing algorithms. In this case, the proposed steps are specific for defect detection using active thermography. The framework is divided in four steps: acquisition, enhancement, filtering and segmentation. For each step some of the most appropriate methods are selected. In the case of acquisition and enhancement there are well known methods already available in the literature that provide very good results. In the case of acquisition specific stimulation methods, such as optical pulsed thermography or optical step heating thermography are usually used. There are also existing methods for signal enhancement, such as polynomial fitting [10] and pulsed phase thermography [11]. For filtering and segmentation several alternatives are proposed. Finally, the proposed framework is applied to the automatic detection of defects in carbon fiber composites.

\section{Defect detection}

Image processing algorithms are generally broken down into several steps. The first step is the acquisition of the images. Next, there is a pre-processing step designed to reduce the noise and improve the quality of the image. As 
part of this step, there is usually a non-uniform background removal process. Segmentation is usually applied next to divide the image into segments of similar attributes. The goal is to find suspicious regions of interest containing abnormalities. Next, there is a feature extraction step, where features are calculated in order to provide a numerical characterization of the segmented objects. Features extracted from an object aim to characterize the object to be recognized by measurements whose values are very similar for objects in the same category, and very different from objects in other categories. Finally, on the basis of the extracted features there is a classification step where pattern recognition techniques are applied to identify the types of objects.

Over the past few decades, major advances have occurred in each of these steps, and many image analysis techniques have been proposed. However, infrared images have special particularities, such as non-uniformities and blurry edges, which make many traditional image analysis techniques unsuitable [12]. This is why many applications process infrared images using ad hoc algorithms [13].

The general approach used by image processing algorithms can also be applied to infrared images. However, it is necessary to select the most adequate methods for each of the processing steps. They need to be adapted to the particularities of infrared images. The steps of the proposed framework for defect detection and the proposed methods for each of steps are presented in the following subsections. Fig. 1 shows a summary of the steps.

\subsection{Acquisition}

In active infrared thermography, acquisition is carried out at the same time as an external stimulus is being applied to the inspected specimen, causing non-stationary heat flow. The objective is to create thermal contrast on subsurface anomalies, i.e., the defects.

Infrared image acquisition must be carried out using an infrared camera. There are different types depending on the spectral range: long wave infrared cameras, sensitive from 8 to $15 \mu \mathrm{m}$, and middle wave infrared cameras, sensitive from 3 to $5 \mu \mathrm{m}$. In general, middle wave infrared cameras are more frequently used, as they outperform long wave infrared cameras for non-destructive testing [14].

Many different stimulation methods can be applied to create thermal contrast on subsurface anomalies. Most of them can be classified as optical, mechanical or inductive. The most common are based on optical stimulation, which use light to deliver energy to the specimen. The applied stimulation generates heat which propagates as thermal waves from the surface through the specimen. When the thermal waves reach an anomaly they change their propagation rate, producing thermal contrast on the surface immediately above the anomaly.

Two of the most common stimulation methods are optical pulsed thermography and optical step heating thermography. Optical pulsed thermography consists of a short (pulsed) heating of the specimen for a few milliseconds. The temperature decay curve is then recorded with the infrared camera. The presence of defects reduces the diffusion rate, resulting in thermal contrast on the surface of the specimen immediately above the defects. Generally, the pulse is generated with flash lamps. Optical step heating thermography uses a much longer pulse than the previous stimulation method. These pulses can last more than 10 seconds. Both the temperature increase and decay are of interest. Deviations from the temperature evolution of a sound area indicate subsurface anomalies. Generally, this type of stimulation is applied using halogen lamps.

\subsection{Enhancement}

Some subsurface anomalies are so subtle that the signal levels associated to them can be lost in the thermographic data noise. In these cases, the acquired raw thermographic data does not provide very useful information. One possible solution is to apply a post-processing method in order to improve the signal-to-noise content of thermographic data. These methods optimise the location and visualization of defects, greatly improving the defect detection rate. The two methods that provide the best results are polynomial fitting and pulsed phase thermography [15].

Polynomial fitting is a common enhancing and filtering method in thermographic inspection. This method fits the temperature time history of each pixel to an n-degree polynomial. Then, the resulting polynomials are differentiated to produce first and second derivatives. In the case of pulsed thermography, the temperature time history of each pixel is previously converted to a logarithmic domain to improve the fit. This enhancing method is commonly called Thermographic Signal Reconstruction (TSR).

Another useful enhancing method is based on the phase of the Discrete Fourier Transform. In order to calculate the phase of the thermographic data, the temperature time history of each pixel is transformed into the frequency domain using the Discrete Fourier Transform. This method applied to optical pulsed thermography is called pulsed phase thermography (PPT) and combines the advantages of modulated and pulse infrared thermography.

\subsection{Filtering}

Thermographic data is greatly affected by noise, by the non-uniform properties of the surface of the material being inspected, and also by the uneven heat generated by the optical stimulus. Part of the non-uniformities are canceled out by the enhancement step but not all. Thus, it is necessary to apply a filtering step.

Filtering is generally applied in two phases: noise filtering and non-uniform background removal. Noise filtering is usually applied by convolving the image with a low-pass filter mask. The most common mask is the Gaussian mask, 
which comprises elements determined by a Gaussian function. Another possible approach is a non-linear filter, such as the median, which preserves image detail more accurately. Non-uniform background removal is usually applied by removing the background intensity level from the image. The most common methods are the top-hat filter [16] and the median [17].

The proposed method for filtering is a modified morphological enveloping filtering process [18]. This filter can be used both for noise removal and for non-uniform background removal. Also, in the case of thermographic data it provides much better results. The filter is applied to the row and column profiles of the images independently. The first step of the filter is to roll a sphere above the signal in order to calculate the top envelope. The sphere is rotated around the first point of the intensity profile until it touches another point. The tangent of the sphere is calculated and the process continues from that point.

Two envelopes are created: a top envelope and a bottom envelope. The top envelope is created using a sphere rolling above the signal. The bottom envelope is created by rolling a sphere below the top envelope. This creates a smoothed envelope that represents the background of the profile. Finally, the smoothed top envelope is subtracted from the signal, removing the noise below the signal and also the background. The proposed filtering method assumes that defect-free regions extend over a significant portion of the inspection images.

Fig. 2 shows an example. Fig. $2 \mathrm{~b}$ shows a row profile of the image shown in Fig. 2a. The top envelope is shown in Fig. 2c. Then, the bottom envelope is calculated, as can be seen in Fig. 2d. This signal represents the background of the intensity profile. In order to remove the background from the intensity profile, the smoothed top envelope is subtracted from the signal. The resulting profile can be seen in Fig. $2 \mathrm{e}$, and the resulting image can be seen in Fig. $2 f$.

This approach not only performs robustly, it also leaves the amplitude of the regions with the defects unmodified. That is, it is not averaged with neighbor pixels. Thus, removing the background also removes the influence of the zone of the image in which the defect is located without affecting the amplitude of the defect. It preserves significant changes in image intensity and smooths down random noise fluctuations.

\subsection{Segmentation}

Image segmentation divides the image into sets of segments with similar attributes. Segmentation is one of the oldest and most widely studied aspects of computer vision.

In the case of infrared images, standard image segmentation methods, such as thresholding, edge detection, region growing, or watershed, do not provide good results. This is mainly due to the non-uniformities and blurry edges of the thermographic data, present even after filtering. The proposed method for the segmentation of defects is based on a recently developed group of algorithms called active contours [19].

There are many advantages of active contour models over classic image segmentation methods. For example, active contour models can achieve sub-pixel accuracy of object boundaries, and can incorporate prior knowledge, such as shape and intensity distribution. They also provide smooth closed contours that can be used for shape analysis and recognition. Particularly important is their ability to perform in images with intensity heterogeneities, which often occur in real images taken from both the visible and the infrared spectrum.

Active contour segmentation attempts to minimize an energy function by evolving the current contour towards image features. The energy function is defined based on the specific active contour model. The original model is based on three image forces: internal, external and image forces. Internal forces give the model tension and stiffness. External forces come from human operators or initialization procedures. Images forces are driven by image features such as light and dark regions.

Existing active contour models can be categorized into two major classes: edge-based models, and regionbased models. Edge-based models use local edge information to evolve the contour toward the object boundary, using for example an energy function based on a two-dimensional spline curve. Region-based models use an energy function based on a certain region descriptor which guides the motion of the active contour.

The proposed procedure for the segmentation of defects is based on a region-based active contour model in a variational level set formulation [20]. This active contour model defines a region-scalable fitting energy function in terms of a contour, and two fitting functions that locally approximate the image intensities on the two sides of the contour. This segmentation method can be used to segment images with intensity heterogeneity and also with weak object boundaries.

Segmentation using active contours requires the specification of an initial contour. This initial contour can be created in different ways. One possibility is to apply a rough segmentation technique, such as thresholding, and evolve the contour from this initial primitive segmentation [21].

Fig. 3 shows an example of segmentation using active contours. Fig. 3a shows an image with a synthetic defect. The image has been corrupted with Gaussian noise, and the intensity of the defect is not uniform. Global image thresholding using Otsu's method is applied to the image to calculate an initial contour (Fig $3 \mathrm{~b}$ ). The result is a rough segmentation that only detects part of the defect. Then, the segmentation method modifies the contour as can be seen in Fig. 3c-f. The initial contour evolves towards an accurate segmentation of the foreground and background.

\section{Results and discussion}

The proposed procedure has been tested using a carbon fiber composite. Fig. 4 shows the test piece used in the experiments. It is a carbon fiber composite used to build aeronautical structures. The specimen is made of several 
layers of carbon-fiber filled with epoxy, as can be seen in Fig. 5. In the thickest part, the test piece contains 12 layers, whereas in the thinnest part it contains only 3. Each layer is numbered from 1 to 12 . There are four zones, from A to D, with a width of $75 \mathrm{~mm}, 120 \mathrm{~mm}, 165 \mathrm{~mm}$, and $210 \mathrm{~mm}$, respectively. These zones have different thickness. The first one has a thickness of 3 layers, the second one of 6 layers, the third one of 9 layers, and the last one of 12 layers. The defects in the specimen are Teflon and Film types, i.e., inserts of teflon and film at different depths.

Acquisition is based on optical pulsed thermography. The setup consists of two flash lamps used in reflection mode. The flash heating system outputs a heating pulse of approximately 6 kilojoules per few milliseconds.

All the experiments were recorded using a FLIR SC5000 camera. This camera is equipped with a cooled Indium antimonide detector which operates in the 2.5 to $5.1 \mu \mathrm{m}$ waveband. The FLIR SC5000 produces thermal images of $320 \mathrm{x}$ 256 pixels with 12 bits per pixel and a thermal sensitivity of $20 \mathrm{mK}$. Although the camera has a maximum frame rate of $383 \mathrm{~Hz}$, the experiments were recorded at $50 \mathrm{~Hz}$ to reduce the number of acquired images.

Figs. $6,7,8$, and 9 show the results of the experiments. These figures correspond to zones $A$ to $D$ of the test specimen. Thus, Fig. 6 correspond to defects below layer 1, Fig. 7 correspond to defects below layers 3 and 4 , Fig. 8 correspond to defects below layers 5, 6, and 7, and Fig. 9 correspond to defects below layers 7, 8, 9, and 10. For each of these figures there are four images. The first image (subfigure a) is a raw infrared image, the frame with the best SNR. The second image (subfigure b) is the image obtained after enhancement using the phase of the DFT or the second derivative of the polynomial fitting. The third image (subfigure $\mathrm{c}$ ) is the image obtained after filtering. The fourth image (subfigure d) is the image obtained after segmentation. These four images are the results of the four steps of the processed detection framework.

The results show that the proposed detection framework is not able to detect all the defects. However, it is not a failure of the detection process, as the defects could not be detected by an experienced technician either. An acquisition based on optical step heating thermography and with more energy would probably provide better results. However, with the energy used in the experiments a human without previous knowledge of the defects would probably have identified the same defects as the proposed framework. Thus, the detection shown in the results can be considered a success. No false defects are detected, and the missing defects are due to the inability of the non-destructive technique to create enough thermal contrast.

Results also show a strong correlation between the depth of the defect and the detectability. The deeper the defect the harder it becomes to detect. This is a known issue of non-destructive thermographic inspection because the detection of deeper defects requires more energy. For the same depth, Teflon defects are much easier to detect. This is due to the thermal properties of the material, such as thermal conductivity.

\section{Conclusions}

In this work a framework for automatic defect detection using active thermography is proposed. The proposed framework is based on four steps: acquisition, enhancement, filtering and segmentation. The most appropriate methods are selected for each step. In the cases of acquisition and enhancement, existing methods are reviewed. For filtering and segmentation several alternatives are proposed adapted to the specific particularities of the problem.

The performance of the proposed method was evaluated using a real carbon fiber composite specimen with different types of defects at different depths, from 1 to 10 layers. The results indicate that the defect detectability is similar to what a human would obtain using a post-processing method.

The proposed detection framework is not the definitive work on the topic. There are still many issues that need to be solved before the integration of this process in the automation of a production line. One of the most important is the automatic selection of the stimulation and enhancement method. A priori, there is no single stimulation or postprocessing method which maximizes SNR in all scenarios. However, the authors believe the proposed framework is a step forward in the automation of fault detection using active thermography.

\section{ACKNOWLEDGEMENTS}

This work was supported by the Basque Country Regional Ministry of Industry, Innovation, Trade and Tourism under Project NEUTER for grants received from Scientific-Technological Agents integrated in the Basque Science, Technology and Innovation Network.

\section{REFERENCES}

[1] B. G. Dale, A. van der Wiele, and J. van Iwaarden, Managing quality. Blackwell Pub. 2007.

[2] C. Meola and G. M. Carlomagno, "Recent advances in the use of infrared thermography," Measurement science and technology, vol. 15, no. 9, p. R27, 2004.

[3] X. Maldague, "Theory and practice of infrared technology for nondestructive testing". Wiley-Interscience. 2001. 
[4] D. L. Balageas, "Defense and illustration of time-resolved pulsed thermography for NDE," Quantitative InfraRed Thermography Journal, vol. 9, no. 1, pp. 3-32, 2012.

[5] A. Mital, M. Govindaraju, and B. Subramani, "A comparison between manual and hybrid methods in parts inspection," Integrated Manufacturing Systems, vol. 9, no. 6, pp. 344-349, 1998.

[6] C. Ibarra-Castanedo, D. Gonzalez, M. Klein, M. Pilla, S. Vallerand, and X. Maldague, "Infrared image processing and data analysis," Infrared physics \& technology, vol. 46, no. 1, pp. 75-83, 2004.

[7] J. Bozek, M. Mustra, K. Delac, and M. Grgic, "A survey of image processing algorithms in digital mammography," in Recent Advances in Multimedia Signal Processing and Communications, Springer, 2009, pp. 631-657.

[8] I. Smal, M. Loog, W. Niessen, and E. Meijering, "Quantitative comparison of spot detection methods in fluorescence microscopy," Medical Imaging, IEEE Transactions on, vol. 29, no. 2, pp. 282-301, 2010.

[9] A. Garakani, D. J. Michael, and J. Koljonen, "Automated optical inspection apparatus," Patent 564019917-Jun1997.

[10] S. M. Shepard, J. R. Lhota, B. A. Rubadeux, D. Wang, and T. Ahmed, "Reconstruction and enhancement of active thermographic image sequences," Optical Engineering, vol. 42, no. 5, pp. 1337-1342, 2003.

[11] X. Maldague, F. Galmiche, and A. Ziadi, "Advances in pulsed phase thermography," Infrared physics \& technology, vol. 43, no. 3, pp. 175-181, 2002.

[12] Y. Zhou and M. Omar, "Dynamic-template processing for passive thermograms: Applied to automotive stamping split detection," NDT \& E International, vol. 41, no. 8, pp. 582-588, 2008.

[13] R. Heriansyah and S. A. R. Abu-Bakar, "Defect detection in thermal image for nondestructive evaluation of petrochemical equipments," NDT \& E International, vol. 42, no. 8, pp. 729-740, 2009.

[14] I. Jorge Aldave, P. Venegas Bosom, L. Vega González, I. López de Santiago, B. Vollheim, L. Krausz, and M. Georges, "Review of thermal imaging systems in composite defect detection," Infrared Physics \& Technology, vol. 61, pp. 167-175, Nov. 2013.

[15] R. Usamentiaga, P. Venegas, J. Guerediaga, L. Vega, and I. López, "A quantitative comparison of stimulation and post-processing thermographic inspection methods applied to aeronautical carbon fibre reinforced polymer," Quantitative InfraRed Thermography Journal, vol. 10, no. 1, pp. 55-73, 2013.

[16] H. Zheng, L. X. Kong, and S. Nahavandi, "Automatic inspection of metallic surface defects using genetic algorithms," Journal of materials processing technology, vol. 125, pp. 427-433, 2002.

[17] R. Usamentiaga, P. Venegas, J. Guerediaga, L. Vega, and I. López, "Non-destructive inspection of drilled holes in reinforced honeycomb sandwich panels using active thermography," Infrared Physics \& Technology, 2012.

[18] R. Usamentiaga, P. Venegas, J. Guerediaga, L. Vega, and I. López, "Automatic detection of impact damage in carbon fiber composites using active thermography," Infrared Physics \& Technology, 2013.

[19] M. Kass, A. Witkin, and D. Terzopoulos, "Snakes: Active contour models," International journal of computer vision, vol. 1, no. 4, pp. 321-331, 1988.

[20] C. Li, C.-Y. Kao, J. C. Gore, and Z. Ding, "Minimization of region-scalable fitting energy for image segmentation," Image Processing, IEEE Transactions on, vol. 17, no. 10, pp. 1940-1949, 2008.

[21] R. Usamentiaga, J. Molleda, D. Garcia, and F. Bulnes, "Monitoring Sintering Burn-Through Point Using Infrared Thermography," Sensors, vol. 13, no. 8, pp. 10287-10305, Aug. 2013.

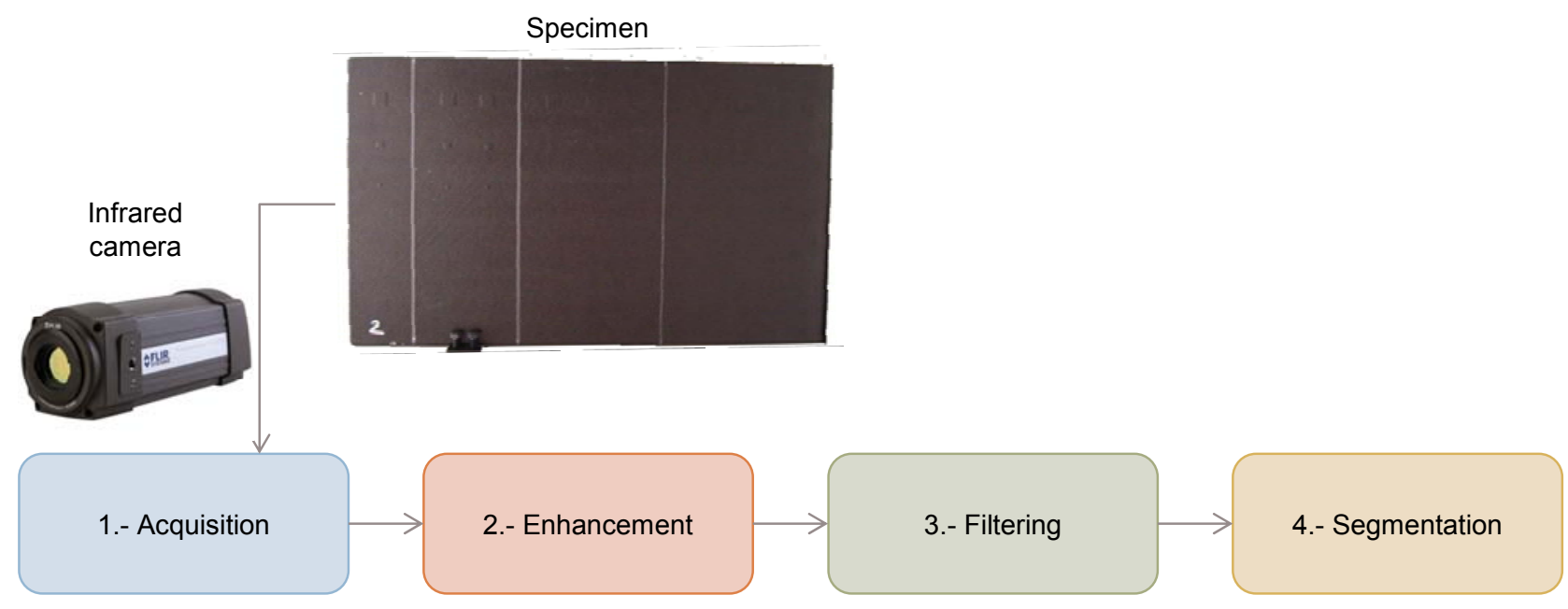

Fig. 1. Steps of the proposed detection framework 


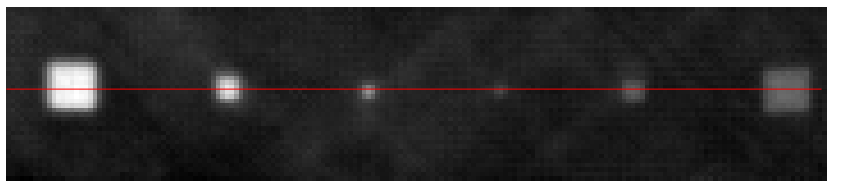

(a)

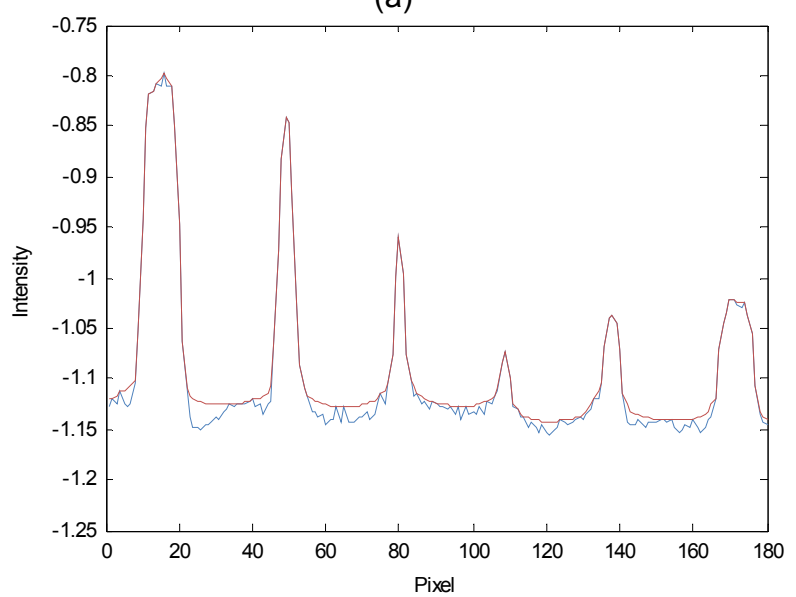

(c)

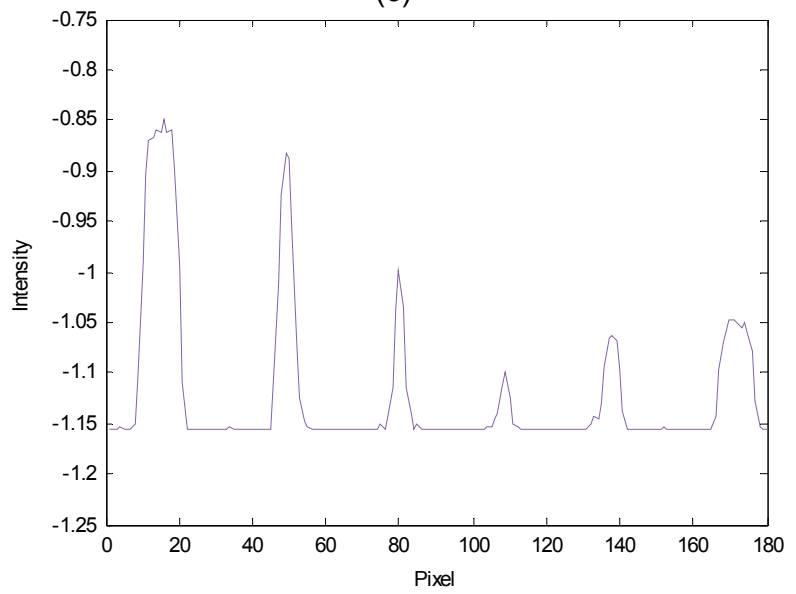

(e)

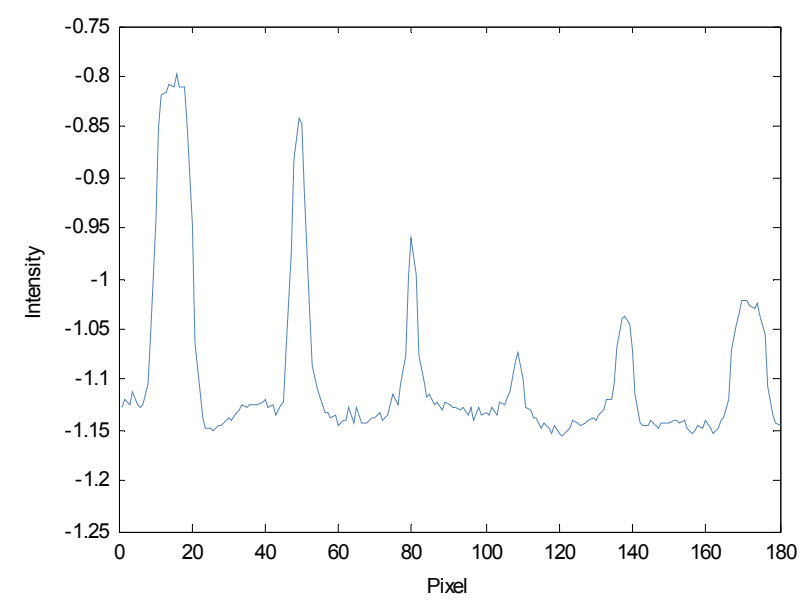

(b)

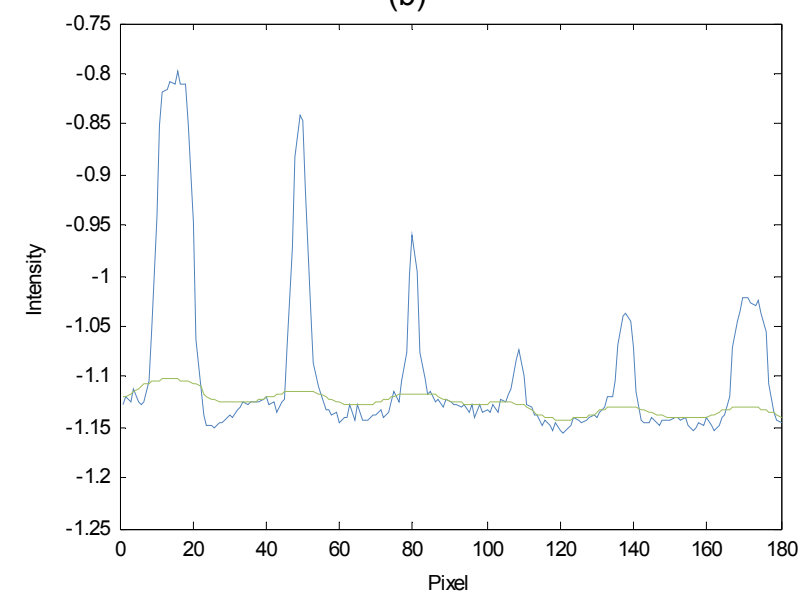

(d)

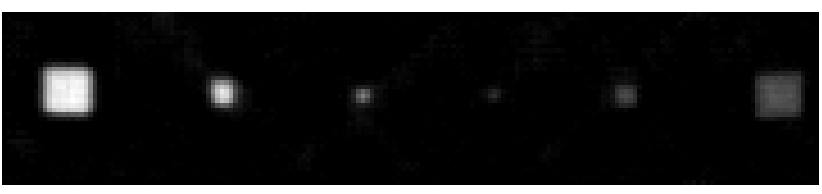

(f)

Fig. 2. Filtering. (a) Image, (b) Intensity profile, (c) Top envelope, (d) Bottom envelope, (e) Filtered profile, ( $f$ ) Filtered image 


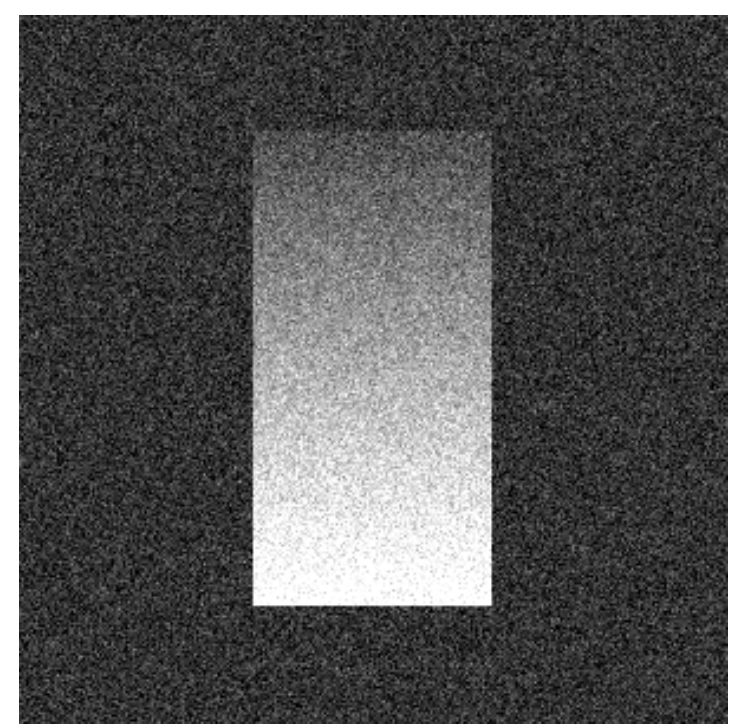

(a)

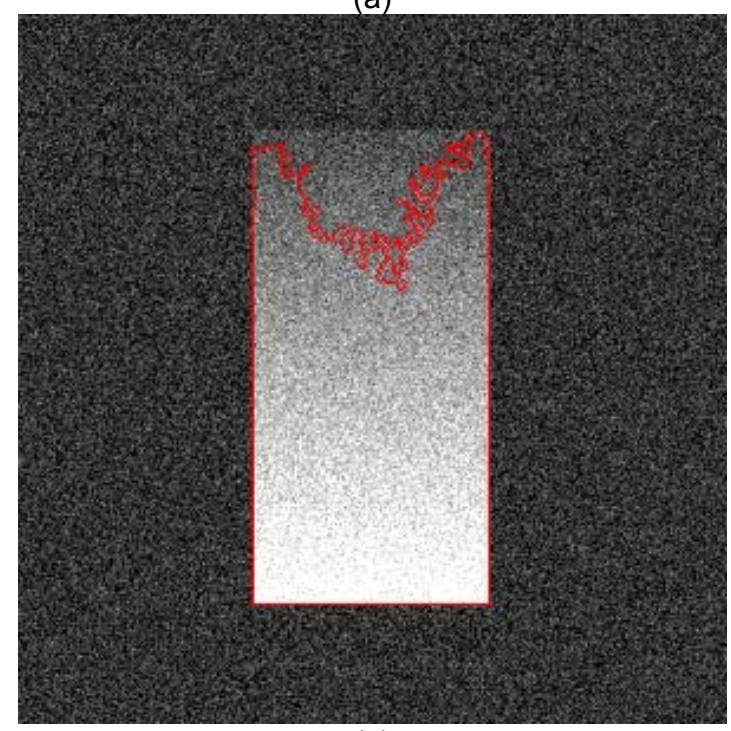

(c)

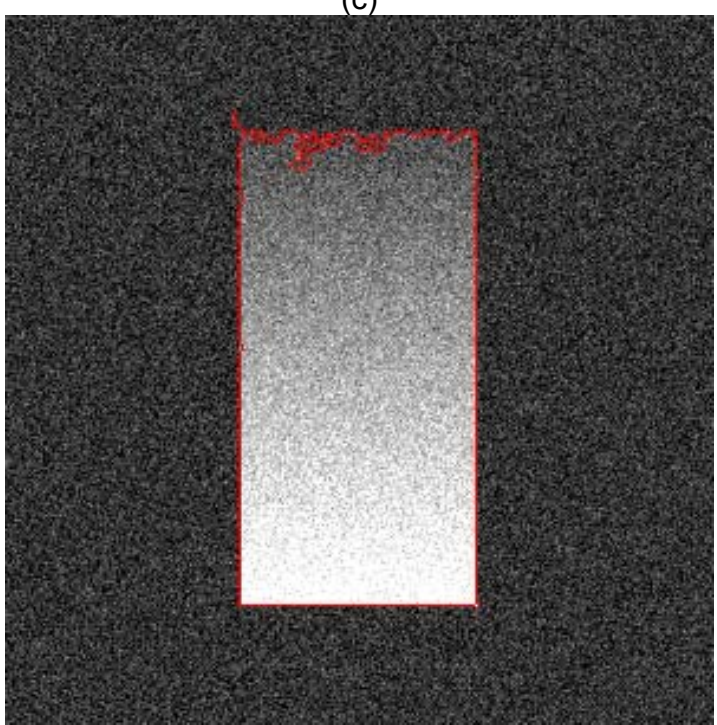

(e)

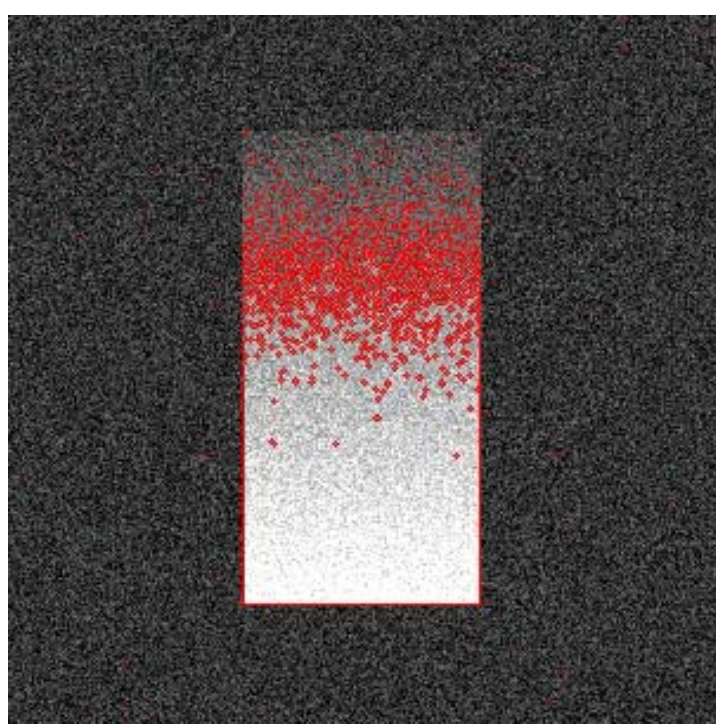

(b)

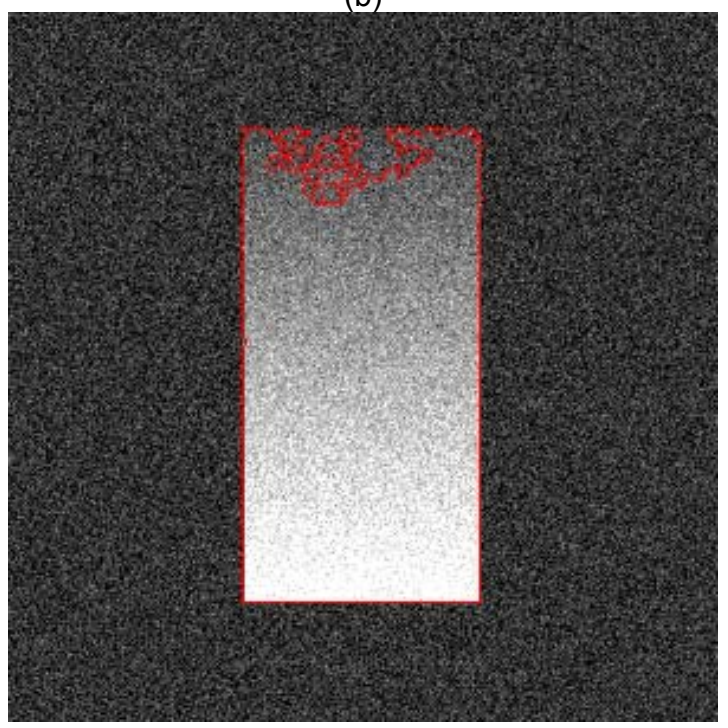

(d)

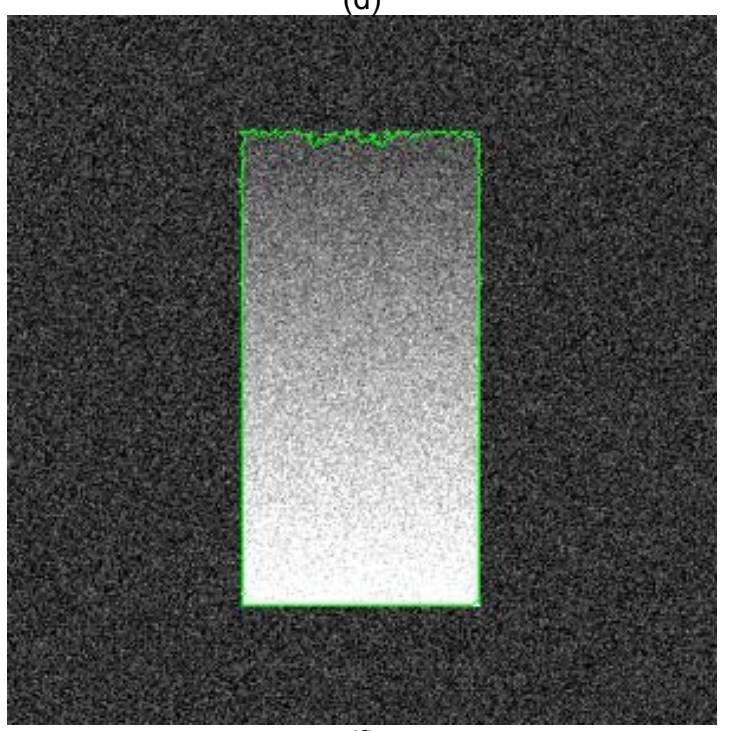

(f)

Fig. 3. Segmentation with active contours. (a) Image, (b) Thresholding, (c) After 5 iterations, (d) After 10 iterations, (e) After 20 iterations, ( $f$ ) After 40 iterations 


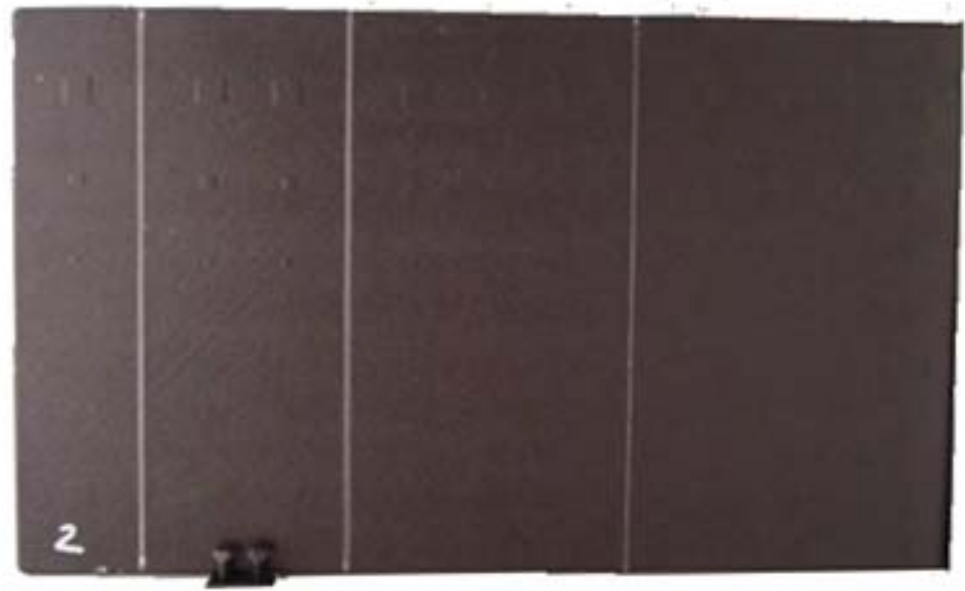

Fig. 4. Test piece

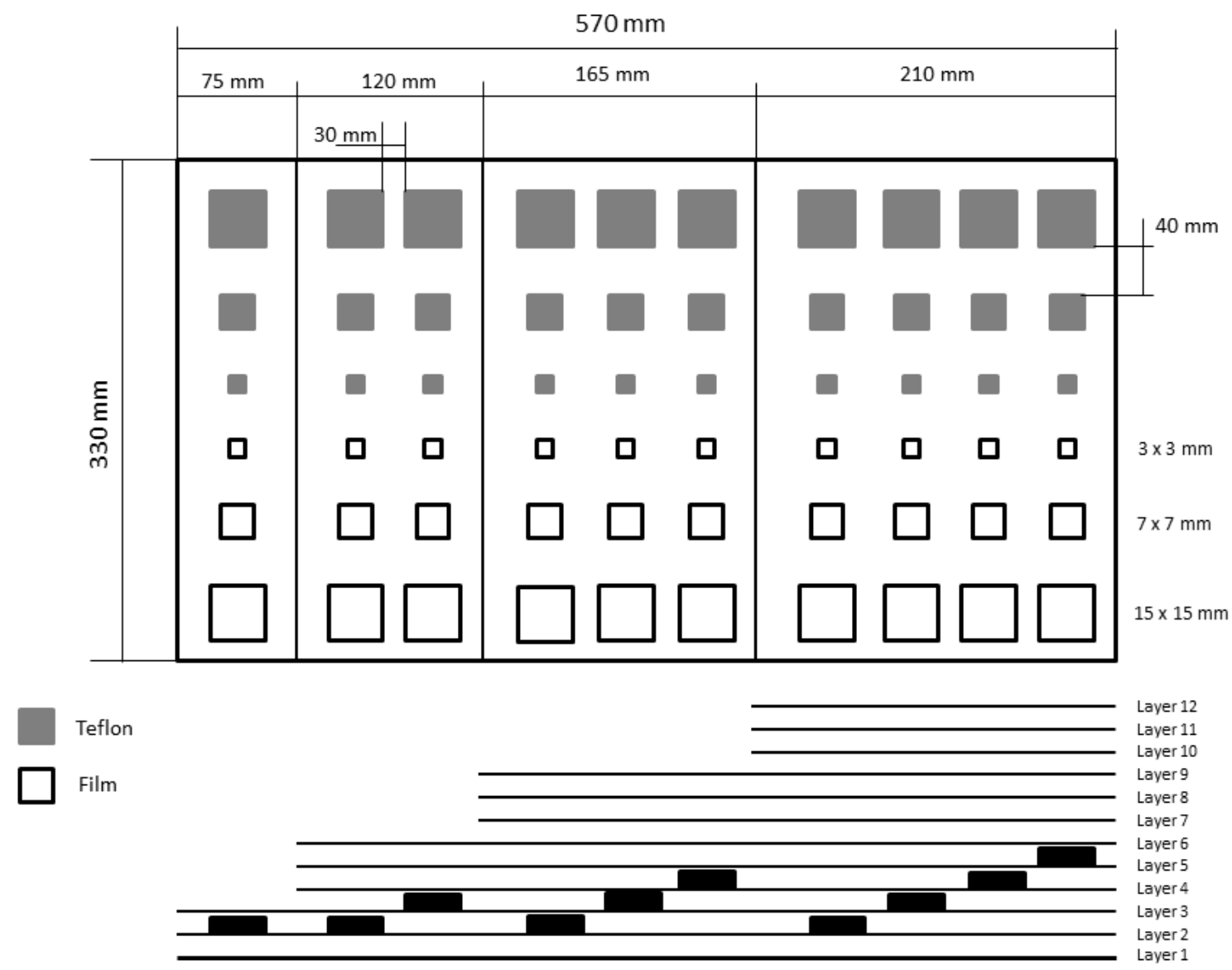

Fig. 5. Defect map 


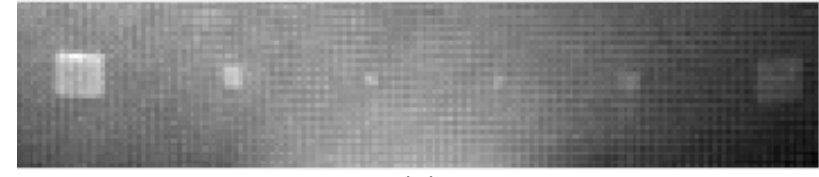

(a)

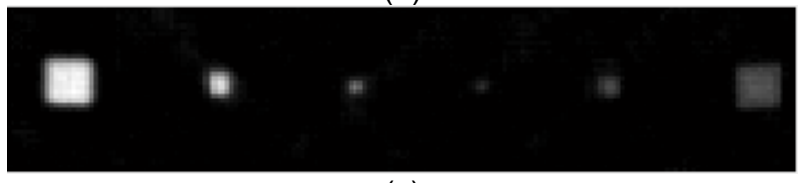

(c)

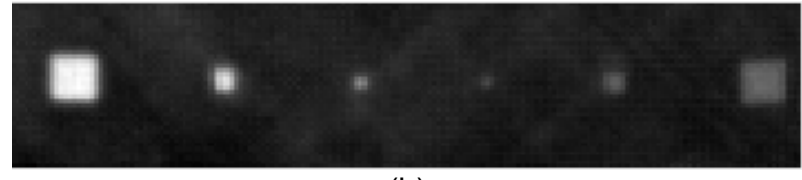

(b)

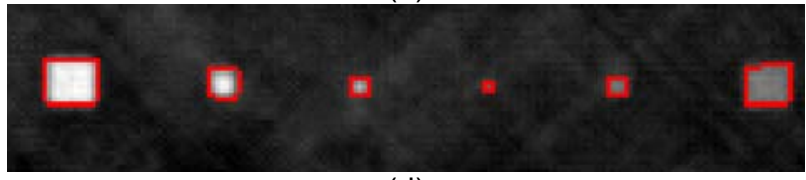

(d)

Fig. 6. Results with defects below layer 1. (a) Raw infrared image, (b) Enhancement, (c) Filtering, (d) Segmentation

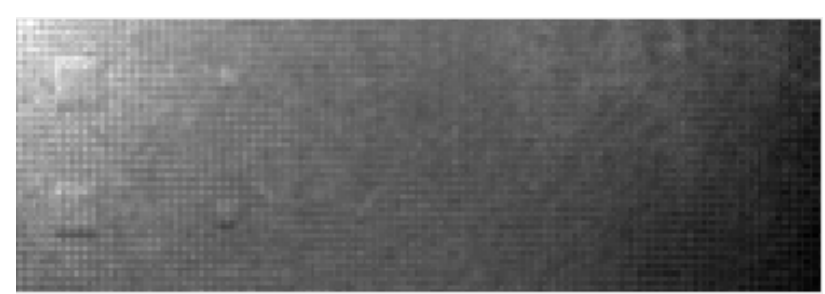

(a)

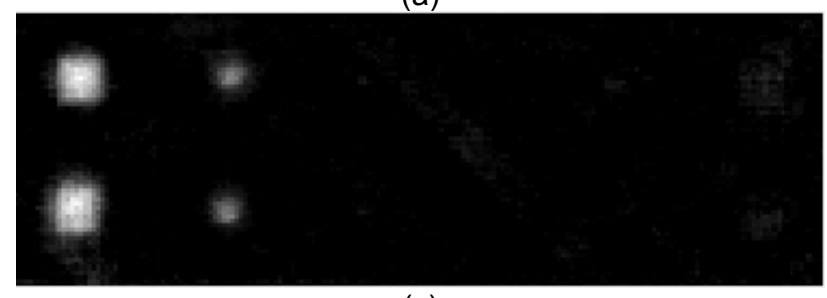

(c)

Fig. 7. Results with defects below layers 3 and 4. (a) Raw infrared image, (b) Enhancement, (c) Filtering, (d) Segmentation

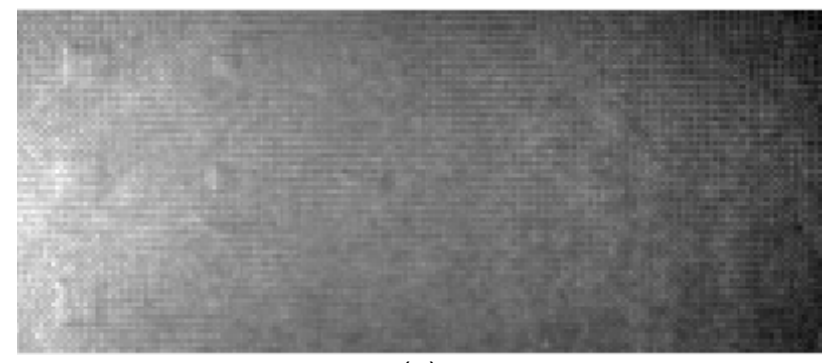

(a)

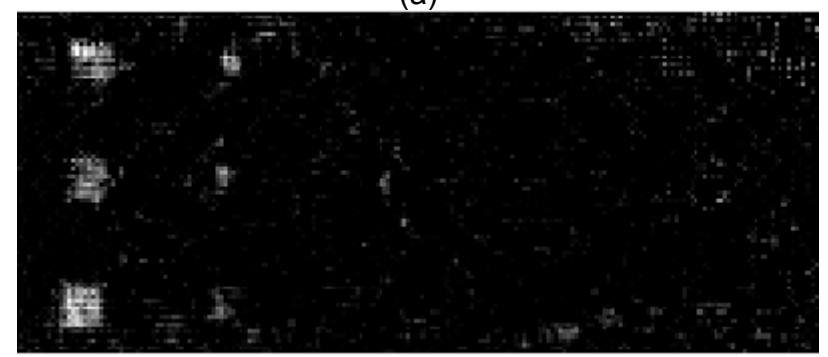

(c)

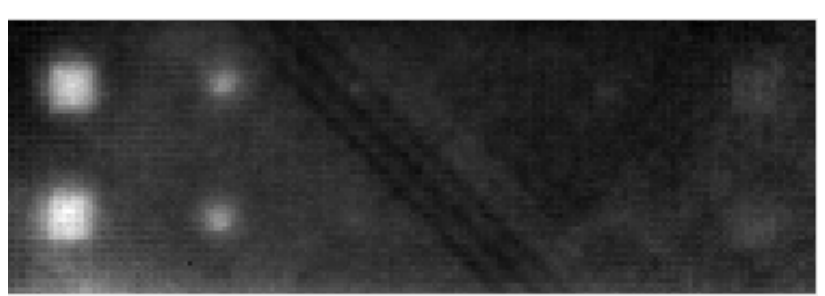

(b)

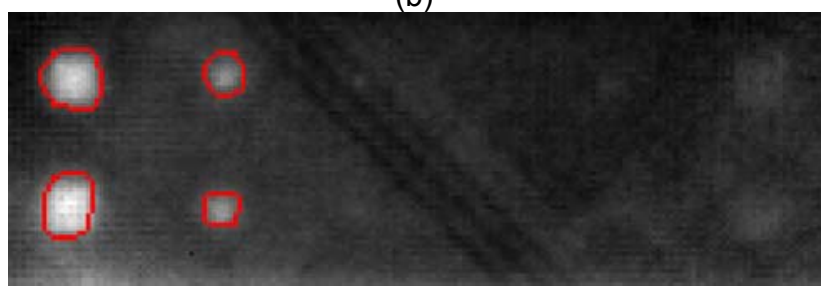

(d)

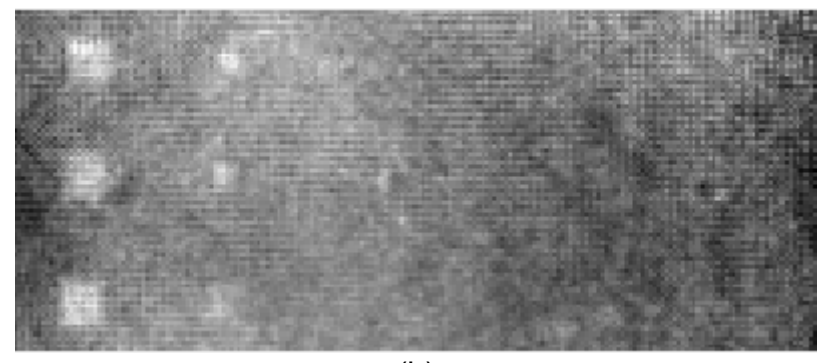

(b)

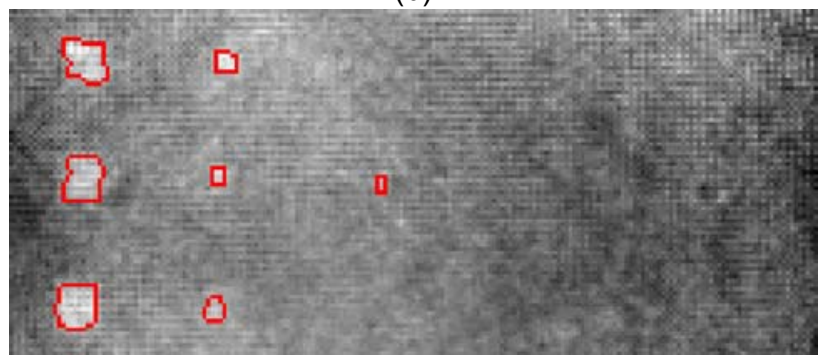

(d)

Fig. 8. Results with defects below layers 5, 6, and 7. (a) Raw infrared image, (b) Enhancement, (c) Filtering, (d) Segmentation 


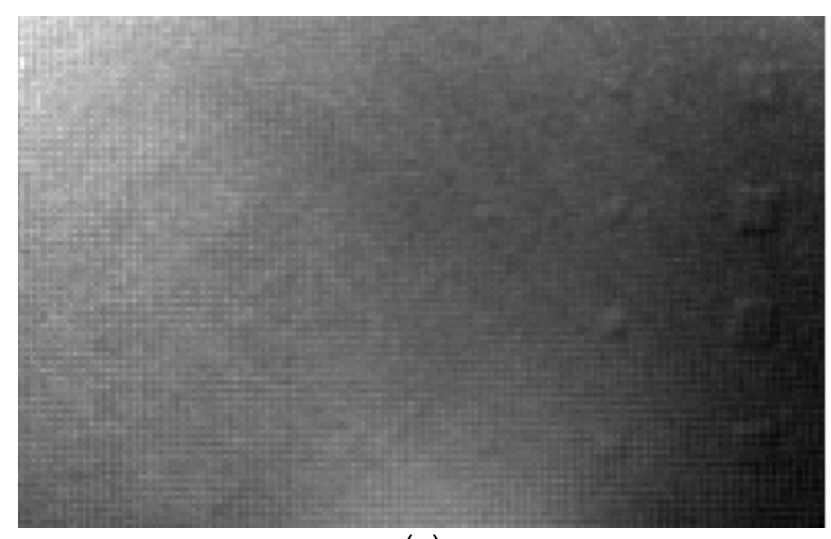

(a)

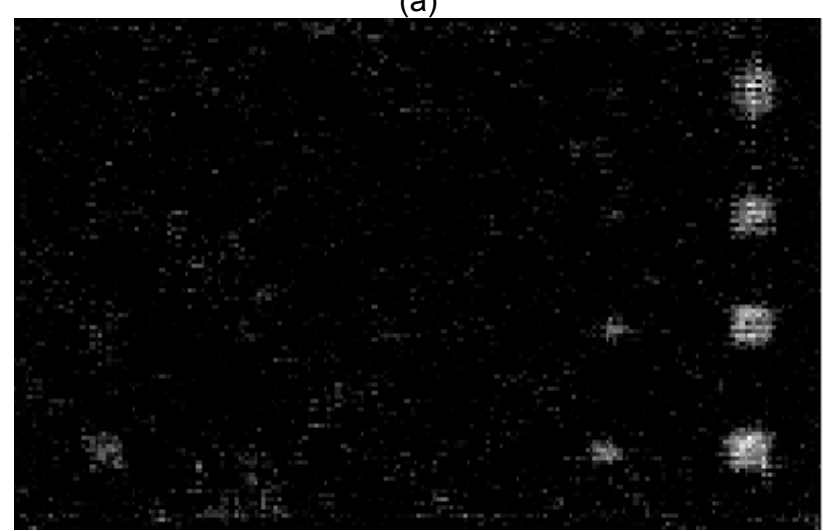

(c)

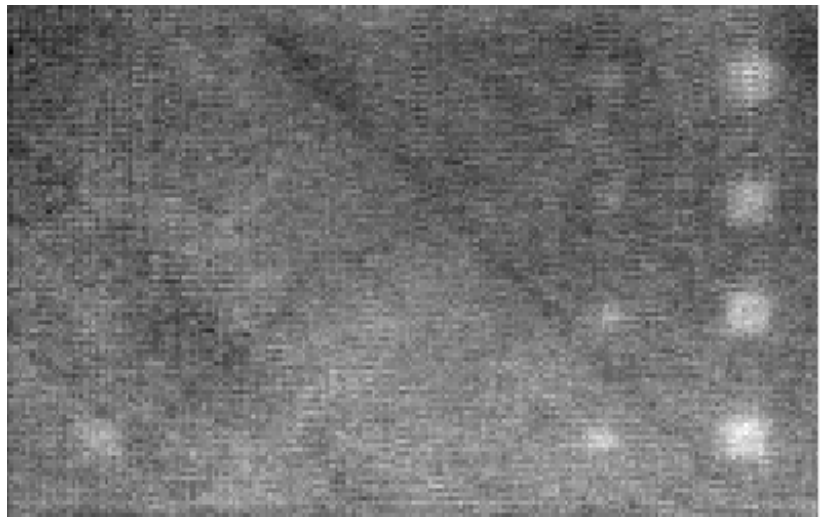

(b)

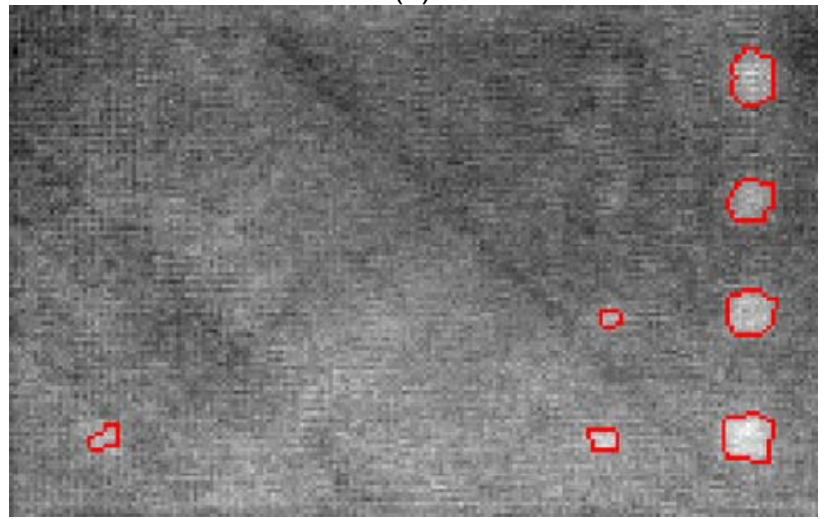

(d)

Fig. 9. Results with defects below layers 7, 8, 9, and 10. (a) Raw infrared image, (b) Enhancement, (c) Filtering, (d) Segmentation 